\title{
Evaluation of Crystal Lattice Rotation around a Stress-Induced Twin in a Step- Graded SiGe / Si (110) Using STEM Moiré Observation and its Image Analysis
}

\author{
Junji Yamanaka ${ }^{*}$, Chiaya Yamamoto ${ }^{1}$ Mai Shirakura ${ }^{1}$, Kosuke O. Hara ${ }^{2}$, Keisuke Arimoto ${ }^{2}$, \\ Kiyokazu Nakagawa ${ }^{2}$, Akimitsu Ishizuka $^{3}$, and Kazuo Ishizuka ${ }^{3}$ \\ 1. Center for Instrumental Analysis, University of Yamanashi, 4-3-11 Takeda, Kofu, JAPAN \\ 2. Center for Cryst. Sci. \& Tech., University of Yamanashi, Miyamaecho, Kofu, JAPAN \\ 3. HREM Research Inc., 14-48 Matsukazedai, Higashimastuyama, JAPAN \\ *Corresponding author: jyamanak@yamanashi.ac.jp
}

Elastic lattice strain strongly affects electric properties of semiconductor materials. For example, a strained $\mathrm{Si}$ exhibits higher carrier-mobility than an ordinary Si. SiGe virtual substrates are commonly used to produce the strained $\mathrm{Si}[1,2]$. The key point is that the SiGe lattice must be relaxed, otherwise it is impossible to produce the strained $\mathrm{Si}$ on the SiGe. Therefore, it is important to analyze lattice strain of both the strained Si and the SiGe virtual substrate. Raman spectroscopy and X-ray reciprocal mapping (XRM) are useful to analyze lattice strain when we don't need nm-order spatial resolution. On the other hand, TEM related methods such as high resolution TEM/STEM images and nano-beam electron diffraction are useful in the case that the spatial resolution is important, although these TEM related methods are not suitable to acquire the data from micrometer order area. Recently a new method to analyze lattice strain was reported by some researchers $[3,4]$. The new method uses a STEM moiré, a moiré between scanning lines of STEM and crystal lattice. This method can acquire data from a wide area with good spatial resolution. We also reported the usage of this technique, especially from the viewpoint of without using Cs-corrected machines $[5,6]$. We reported our feasibility study about a slight change of lattice spacing of the SiGe due to Ge composition change [6]. In this study we focused on the local lattice rotation around a stress-induced twin in the SiGe.

The same specimen which we had investigated lattice spacing was used for this study so we show just the essence of the specimen preparation procedure [6]. A Si/SiGe/Si (110) hetero-structure was produced using MBE. The SiGe part was composed of 1 thick $(440 \mathrm{~nm})$ layer and 9 thin $(40 \mathrm{~nm}$ each) layers. We evaluated the Ge composition of the thick SiGe layer was 22 at $\%$ using XRM. The Ge composition of the thin layers were estimated to be $19.8,17.6,15.4,13.2,11, \ldots, 4.4,2.2$ at $\%$. The foil for the STEM observation was fabricated by using FIB with $\mathrm{Ga}^{+}$ions acceleration voltage of 40 and $10 \mathrm{kV}$. A fieldemission type STEM (FEI Tecnai Osiris) without any Cs correctors was utilized with an acceleration voltage of $200 \mathrm{kV}$. Commercially available software sMoiré (HREM Research Inc.) was utilized in order to analyze the lattice rotation from the STEM moiré [4].

Figures 1a and 1b are STEM bright field images of the specimen. STEM moiré between the scanning lines (scan period is about $0.311 \mathrm{~nm}$ ) and the $\{111\}$ plane can be clearly seen. There exists a stress-induced twin in the observed area. This stress-induced twin causes the relaxation of the SiGe virtual substrate. The authors published the SiGe strain relaxation mechanism in other places [2]. The STEM moiré fringes in Figures $1 \mathrm{a}$ and $1 \mathrm{~b}$ change their spacing and directions, because of the slight change of the scan directions. (This is a typical phenomenon of moiré.) In each image, moiré fringes change their directions across the stress induced twin, although it is a very precise change. In order to analyze this phenomenon objectively, we carried out an image analysis using the software sMoiré. Figures $\mathbf{2 a}$ and $\mathbf{2 b}$ are the power spectra after the appropriate image treatment of Figures 1a and 1b, respectively. The spots have the information 
of the moiré's spacing and direction (see red rectangles), and it means they have the information of the lattice spacing and direction. The insertions are the enlarged pattern around the spots. It is obvious that each spot splits into two spots. This splitting is interpreted as the result of the SiGe lattice rotation across the stress-induced twin. The estimated value of the lattice rotation was about 0.3 degrees both in the cases of Figures $2 \mathrm{a}$ and $2 \mathrm{~b}$. Further efforts are needed to make this method more reliable, but these data indicates that this method has a potential to analyze very precise lattice strain, not only the change of lattice spacing but also that of lattice rotation.

\section{References:}

[1] Junji Yamanaka et al., Thin Solid Films 508 (2006), p. 103.

[2] Keisuke Arimoto et al., J. Cryst. Growth, 311 (2009), p. 819.

[3] Y. Kondo and N. Endo, Kenbikyo 49 (2014), p. 226, in Japanese.

[4] Akimitsu Ishizuka, Martin Hytch and Kazuo Ishizuka, Microscopy, 66 (2017), p. 217.

[5] Junji Yamanaka et al., J. Mat. Sci. \& Chem. Eng., 5 (2017), p. 102.

[6] Junji Yamanaka et al., J. Mat. Sci. \& Chem. Eng., 6 (2018), p. 8.

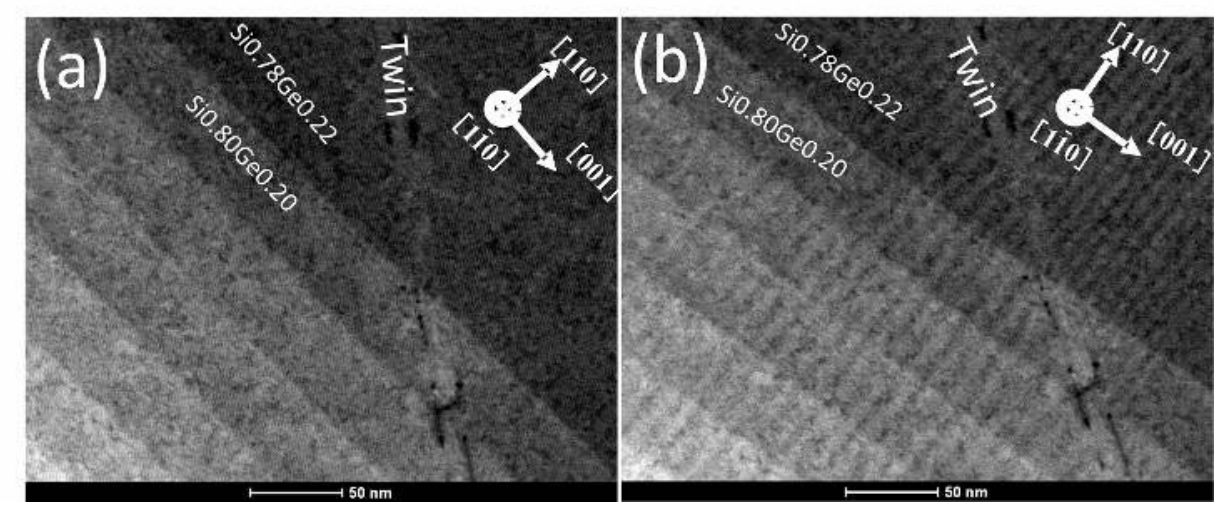

Figure 1. STEM bright field images of the $\mathrm{Si} / \mathrm{SiGe} / \mathrm{Si}$ (110). The observed are was around the interface between the thick SiGe layer and the thin step-graded SiGe layers. STEM moiré fringes can be seen clearly.

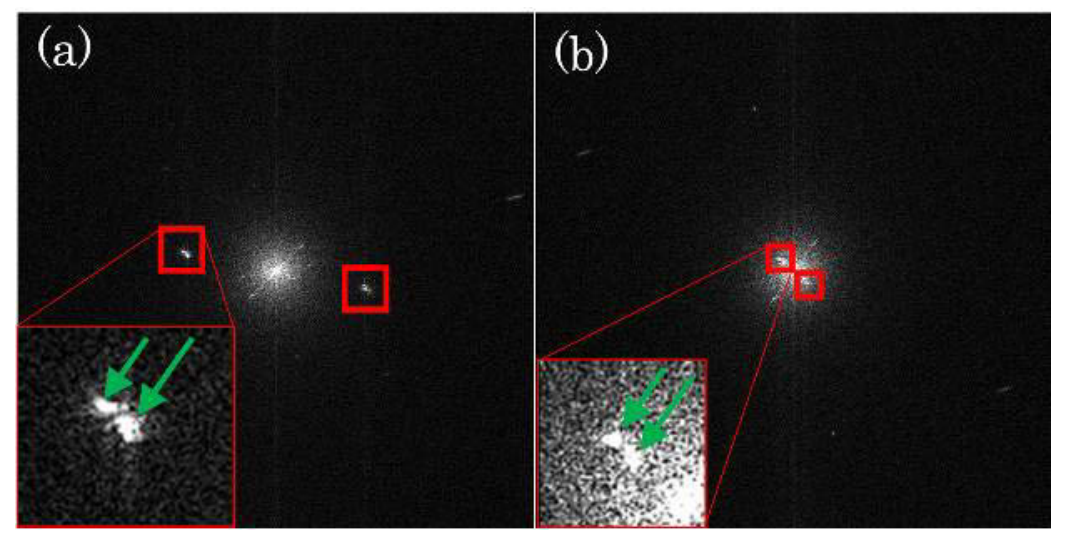

Figure 2. Power spectra corresponding to Figures $1 \mathrm{a}$ and $1 \mathrm{~b}$. Moiré spots can be seen in red rectangles. The insertions are the enlarged pattern. The spots split (see green arrows) and the rotation angle of the SiGe lattice across the twin can be calculated from these data. 